\title{
ULTRAVIOLET Si IV/C IV RATIOS FOR Be STARS
}

\author{
KARL G. HENIZE*, JAMES D. WRAY, S. B. PARSONS, and G. F. BENEDICT \\ Dept. of Astronomy, University of Texas, Austin, Tex., U.S.A.
}

\begin{abstract}
The intensities of the very strong lines of $C$ IV $\lambda 1549 \AA$ and Si IV $\lambda \lambda 1394,1403 \AA$ observed in spectra obtained with Skylab experiment S019 provide a sensitive discrimination of spectral type between B0 and B2. Eye estimates of the Si IV/C IV ratio are tabulated for $33 \mathrm{~B} 0-\mathrm{B} 2$, class III-V stars of which 11 are emission-line stars. Seven of the emission-line stars show significantly smaller ratios than normal stars of the same MK class. The most outstanding examples are $60 \mathrm{Cyg}, o$ Pup, $\eta$ Cen, and $\iota$ Ara.
\end{abstract}

\section{Introduction}

During this symposium Dr Slettebak has already reviewed reasons why it is difficult to assign firm MK classes to the rapidly rotating Be stars, and Dr Heap has noted how data on ultraviolet $\mathrm{C}_{\mathrm{IV}}$ and $\mathrm{Si}$ IV line intensities may be useful in providing improved classifications of such stars. This subject has also been on our minds as we carry out the analysis of ultraviolet spectra obtained with Skylab experiment S019. A preliminary survey of the behavior of the CIV and Si IV lines in early type main sequence stars (Henize et al., 1975) shows that the Si IV/C IV ratio varies dramatically from a value of about 10 at $B 2$ to a value of about $\frac{1}{4}$ at $B 0$. This paper presents a more detailed study of the variation of the $\mathrm{Si}$ IV/C IV ratio as a function of spectral type for 33 B0-B2 stars of which 11 are Be stars.

\section{The Observations}

The instrumentation with which these data were obtained is described by Henize $e t$ al. (1975) and by O'Callaghan et al. (1976). The basic instrument is an objectiveprism spectrograph with a $15-\mathrm{cm}$ aperture and a $4^{\circ}$ prism of $\mathrm{CaF}_{2}$ giving resolutions of $2 \AA$ and $12 \AA$ at wavelengths of $\lambda 1400$ and $2000 \AA$ respectively.

A total of 400 spectra showing measurable fluxes at $\lambda 1500 \AA$ have been obtained and, of these, roughly 120 show evident absorption or emission lines.

Although these spectra are calibrated so that equivalent widths and flux curves may be derived, all factors entering the calibration are not yet completely analyzed and, as a consequence, the data presented here are based on eye estimates of absorption line intensities. These estimates are affected by the differing dispersions and effective exposures at $\lambda 1400 \AA$ vs $1550 \AA$ and it is not to be expected that they will correspond to ratios of the equivalent widths. Nevertheless they represent a self consistent set of data from which stars showing anomalous behavior may be detected.

Initially, spectra of all stars with spectral types B0-B2 and luminosity classes III-V which showed visible flux at $\lambda 1400 \AA \AA$ were examined. This list of 98 stars yielded 34 stars for which both the $\mathrm{C}$ IV and $\mathrm{Si}$ IV lines are well-exposed and in which reasonably reliable line intensities can be determined. These stars and their Si IV/C IV ratios are

* Astronaut Office, Code TE, NASA Johnson Space Center. 
TABLE I

Si IV $\lambda 1394 / C \operatorname{IV} \lambda \lambda 1548,1551$ ratios for B0-B2 stars

\begin{tabular}{|c|c|c|c|c|}
\hline HD & Desig. & Sp. & $\mathrm{Si}$ IV/C IV & Remarks \\
\hline 3360 & $\zeta$ Cas & B2 IV & $\frac{1}{2}$ & CIV very strong for $B 2$ \\
\hline $5394^{*}$ & $\gamma \mathrm{Cas}$ & B0.5 IVe & 1 & \\
\hline $10516^{*}$ & $\varphi$ Per & B2 Vep & $>2$ & Si IV very weak \\
\hline 34816 & $\lambda$ Lep & B0.5 IV & $\frac{3}{4}$ & \\
\hline 35468 & $\gamma$ Ori & B2 III & 10 & \\
\hline 36512 & $v$ Ori & B0 V & $\frac{1}{8}$ & \\
\hline 36822 & $\varphi^{1}$ Ori & B0.5 IV-V & $\frac{1}{4}$ & \\
\hline $50013^{*}$ & $\kappa \mathrm{CMa}$ & B1.5 IVne & $>4$ & \\
\hline $58978^{*}$ & HR 2855 & B0.5 IVnpe & $<\frac{1}{4}$ & \\
\hline $63462^{*}$ & $o$ Pup & B1 IV:nne & $\frac{1}{3}$ & \\
\hline 75821 & HR 3527 & B0 III & $\frac{1}{6}$ & \\
\hline 79351 & a Car & B2 IV-V & $>4$ & \\
\hline 93030 & $\theta$ Car & B0.5 Vp & 2 & $\mathrm{C}$ IV very weak for $\mathrm{B} 0.5$ \\
\hline 108248 & $\alpha^{1}$ Cru & B0.5 IV & 4 & with $\alpha^{2}$ Cru B1 V \\
\hline 116658 & $\alpha$ Vir & B1 IV & 4 & \\
\hline 120640 & HR 5206 & B2 Vp & $>6$ & \\
\hline $120991^{*}$ & HR 5223 & B2 IIIle & & Si IV very weak for $B 2$ \\
\hline 127381 & $\sigma$ Lup & B2 III & 5 & \\
\hline $127972^{*}$ & $\eta$ Cen & B1.5 Vn & 2 & \\
\hline 132058 & $\beta$ Lup & B2 III & $>6$ & \\
\hline 132200 & $\kappa$ Cen & B2 IV & $>6$ & \\
\hline $135160^{*}$ & HR 5661 & B $0.5 \mathrm{~V}$ & $\frac{1}{2}$ & \\
\hline 143018 & $\pi$ Sco & $\mathrm{B} 1 \mathrm{~V}+\mathrm{B} 2$ & 10 & \\
\hline 147165 & $\sigma$ Sco & B1 III & 4 & \\
\hline 149438 & $\tau$ Sco & B0 V & $\frac{1}{3}$ & \\
\hline 151890 & $\mu^{1}$ Sco & B1.5 IV & 4 & \\
\hline $157042^{*}$ & $\iota$ Ara & B2 IIIne & 1: & UV spectrum unwidened \\
\hline 158408 & $v \mathrm{Sco}$ & B2 IV & 6 & \\
\hline 158926 & $\lambda$ Sco & B1.5 IV & 10 & \\
\hline $173948^{*}$ & $\lambda \mathrm{Pav}$ & B2 II-III & 1: & Si IV very weak; C IV broad, Fe III? \\
\hline 188439 & V819 Cyg & B0.5 IIIp & $\frac{1}{2}$ & $1600-2000$ blends strong \\
\hline $200310^{*}$ & 60 Cyg & B1 Vne & $\frac{1}{2}$ & \\
\hline $214168^{*}$ & $8 \mathrm{Lac}$ & $\mathrm{B} 1 \mathrm{Ve}$ & 1: & with HD 214167 B1.5V; C IV broad, Fe III? \\
\hline 224572 & $\sigma$ Cas & B1 V & 4 & \\
\hline
\end{tabular}

* Emission-line star.

listed in Table I. Since the Si IV doublet is well resolved in our spectra and the C IV doublet is not, the ratio given is defined as Si IV $\lambda 1394 / C_{\text {IV }} \lambda \lambda 1548,1551$. The spectral types in Table 1 are derived from Hiltner et al. (1969) and Lesh (1968).

\section{Discussion}

The data of Table I are displayed in Figure 1. For the non-emission line stars there is a clear cut trend for the $\mathrm{Si}$ IV/C IV ratio to increase from about $\frac{1}{5}$ at $\mathrm{B} 0$, through 4 at $\mathrm{B} 1$, to roughly 8 or 10 at $\mathrm{B} 2$. The emission-line stars, on the other hand, show 


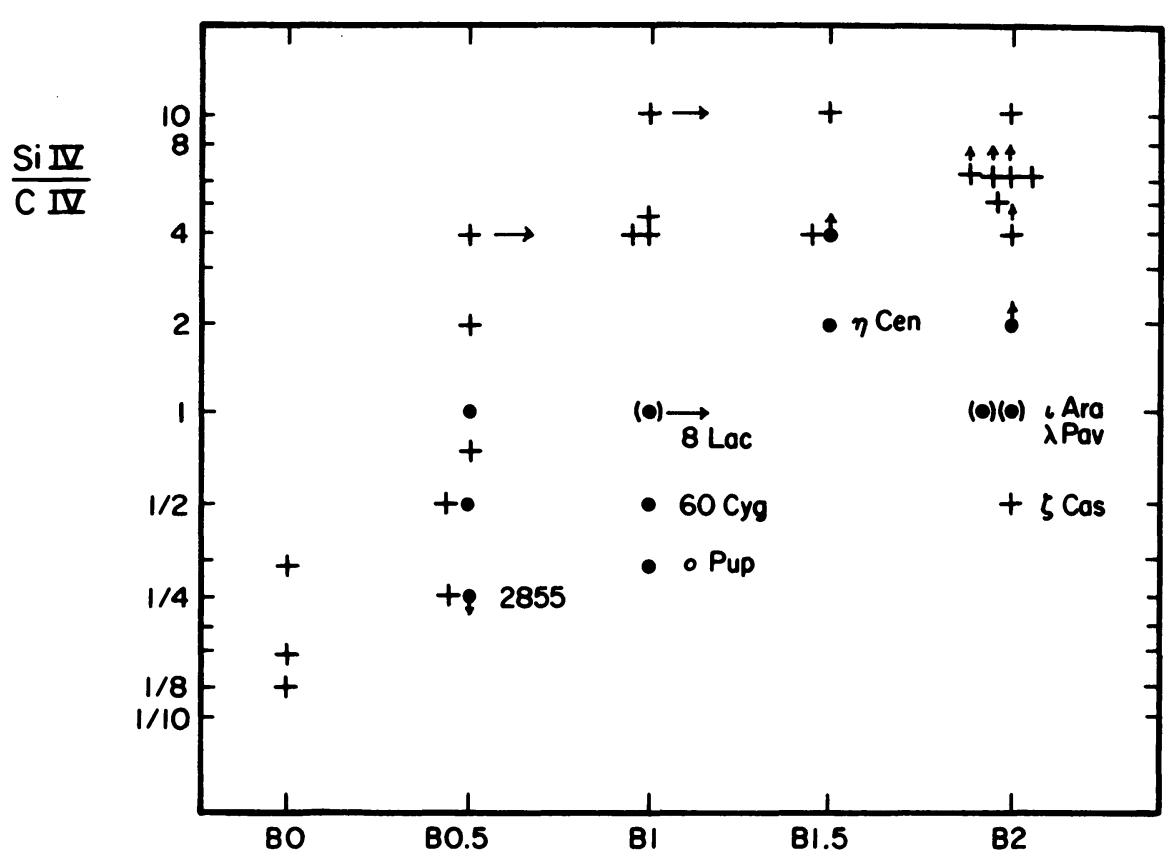

Fig. 1. The ratio Si IV $\lambda 1394 \AA / C$ IV $\lambda \lambda 1548,1551 \AA$ plotted as a function of spectral type. Crosses indicate non-emission-line stars; dots indicate emission-line stars. Parentheses indicate uncertainty in the $\mathrm{Si} / \mathrm{C}$ ratio. $\uparrow$ indicates values which are lower limits of the ratio. $\rightarrow$ indicates that the spectrum is blended with one of slightly later type.

generally smaller ratios than the non-emission-line stars. In particular, seven of the eleven emission-line stars show ratios significantly less than those for normal stars. Of these, four stars (60 Cyg, o Pup, $\eta$ Cen, and $\iota$ Ara) have a C IV intensity conspicuously greater than that of the normal stars of the same spectral class. Two of the remaining stars, $\lambda$ Pav and HR 2855, are peculiar in that the Si Iv lines are unusually weak for their spectral class. This peculiarity is also evident in $\varphi$ Per and HR 5223.

For the four stars in which C IV is enhanced the data in Figure 1 suggest that they are hotter than the MK classes indicate. The rough calibration of the $\mathrm{Si} / \mathrm{C}$ ratio provided in Figure 1 suggests spectral classes of B0.2, B0.5, B0.5, and B0.7 for $o$ Pup, $60 \mathrm{Cyg}$, ` Ara, and $\eta$ Cen, respectively, assuming them to be main sequence stars. However, the $\mathrm{Si} / \mathrm{C}$ ratio is also correlated to luminosity and at spectral class $\mathrm{B} 1$ the ratio ranges from about 0.1 for main sequence stars to about 1 for supergiant stars (see Figure 3d of Henize et al., 1975). Thus the enhancement of C IV may also be due to a lower than expected surface gravity. The $\mathrm{Si} / \mathrm{C}$ ratio in $60 \mathrm{Cyg}$, for example, is also compatible with a classification of B1 II. Since these stars may be expected to show incipient shell absorption and since shell spectra generally show high luminosity characteristics, this is the more attractive of the two possibilities.

The suggestion that the enhancement of CIV absorption may be due to an incipient absorption shell leads to the further question as to whether these four stars show any indication of the extensive blends of weak lines in the $\lambda 1600-2000 \AA$ 
region which are evident in spectra of the advanced shell stars $48 \mathrm{Lib}$ and $\zeta$ Tau and also in many of the $\mathrm{O}$ and early B-type supergiants (see Figure 3a, Henize et al., 1975). A weak indication of such lines is present in $o$ Pup and 60 Cyg but in $\iota$ Ara and $\eta$ Cen this region of the spectrum is over-exposed. It should be noted in passing that these blended shell (or supergiant) absorption features are strong in the star V819 Cyg. There is also a suspicion of weak emission present on the redward edge of $\mathrm{C}$ IV. Thus the ultraviolet spectrum of V819 Cyg is more like that of a B0 or B1 supergiant than that of a B0.5 III star.

The star $\zeta$ Cas also shows enhanced $C$ iv even though it is not an emission-line star. The spectral class suggested by the $\mathrm{Si} / \mathrm{C}$ ratios in Figure 1 is $\mathrm{B} 0.5$. The peculiarity of $\zeta$ Cas is further accentuated by the fact that it is an MK standard (Morgan and Keenan, 1973). The CIV anomaly brings into question whether or not this star is a reliable standard star and further investigation of abundances in this star would seem to be warranted.

One further possibility for explaining $C$ IV enhancement should be mentioned; i.e. the possibility that the C IV line is severely blended with Fe III as is suggested by Peytremann (1975) for all stars cooler than $30000 \mathrm{~K}$. However, in the five stars discussed above the $C_{\text {IV }}$ absorption is sharp and distinct and there is little doubt that it is attributable to C IV. A broad blending of weak lines in the $\lambda 1500$ to $1600 \AA$ region which probably corresponds to the blend studied by Peytremann is visible in many B1 and B2 stars on our plates, but at the resolution of the S019 spectra, it is not easily confused with the $C_{\text {IV }}$ line. The question as to why the empirical data do not agree well with theory is a matter of interest and will be the subject of further study in the S019 spectra.

Two stars in Table $\mathrm{I}, \lambda$ Pav, and $8 \mathrm{Lac}$, do show a somewhat diffuse $\mathrm{C}$ IV line and in this instance it might be suspected that the feature is seriously blended with Fe III. If so, then their $\mathrm{Si} / \mathrm{C}$ ratio is somewhat greater than is indicated in Figure 1. This would remove both stars from the anomalous group of stars so far as the $\mathrm{Si} / \mathrm{C}$ ratio is concerned. However, the fact that $\lambda$ Pav (together with HR 2855, $\varphi$ Per, and HR 5223) shows abnormally weak Si IV bears further consideration. Inspection of Figure 3 of Henize et al. (1975) suggests that the ultraviolet spectra of these stars are more like those of B3 stars in which the Si IV and C IV have almost completely disappeared. The reason for such a trend of misclassification is difficult to understand, however, since in rapidly rotating stars the main effect is to obscure the weak lines required to establish a class of $\mathrm{B} 0$ or $\mathrm{B} 1$ and to enhance the strength of helium. Both effects tend to lead to a classification of B2 and B3 for rapid rotators even though they may be considerably hotter.

\section{References}

Henize, K. G., Wray, J. D., Parsons, S. G., Benedict, G. F., Bruhweiler, F. C., Rybski, P. M., and O'Callaghan, F. G.: 1975, Astrophys. J. Letters 199, L119.

Hiltner, W. A., Garrison, R. F., and Schild, R. E.: 1969, Astrophys. J. 157, 313.

Lesh, J. R.: 1968, Astrophys. J. Suppl. Ser. 17, 371.

Morgan, W. W. and Keenan, P. C.: 1973, Ann. Rev. Astron. Astrophys. 11, 29.

O'Callaghan, F. G., Henize, K. G., and Wray, J. D.: 1976, Appl. Opt., in press.

Peytremann, E.: 1975, Astron. Astrophys. 39, 393. 


\section{DISCUSSION}

Noerdlinger: I can comment on the peculiarity in Si IV you mentioned for $\zeta$ Pup. About a year or more ago Tom Hewitt and I published in Astrophys. J. 188, 315 (1974) a theoretical discussion of the formation of P Cyg resonance lines in the case of close doublets. The absence of a red absorption component in $\zeta$ Pup can be explained by filling-in by the redward emission from the blue component, provided the density is high enough. In fact, doublet structure can be used in this way to diagnose the density in a mass outflow. For Si IV lines, one needs the greatest expansion velocity to be $2000 \mathrm{~km} \mathrm{~s}^{-1}$ or more. That value may be reasonable for $\zeta$ Pup, which has a very rapid wind. 